\section{Psoriasis und Ekzem: antigenspezifische T-Zellen als Zünder?}

\begin{abstract}
Psoriasis wird durch eine von $\mathrm{TH}_{1}$-Zellen dominierte Immunantwort ausgelöst, bei der atopischen Dermatitis überwiegen TH2-Zellen. Ein gemeinsames Auftreten der Erkrankungen ist daher selten. Forscher aus München haben drei Patienten mit beiden Dermatosen untersucht und wichtige Erkenntnisse zur Pathogenese gewonnen.
\end{abstract}

A uch wenn der klinische Verlauf von Psoriasis und atopischer Dermatitis selten überlappten, gab es bei allen drei Patienten Phasen, in denen beide Krankheiten aktiv waren. Zu diesen Zeitpunkten entnommene Hautbiopsate zeigten histologisch die typischen Eigenschaften der jeweiligen Krankheit. In Psoriasisläsionen fand sich zudem eine große Zahl von TH1- und TH17-Zellen und dementsprechend eine verstärkte Sekretion von $\gamma$-Interferon und Interleukin-17 (IL-17). Ekzemregionen enthielten dagegen vor allem TH2- und TH22-Zellen und produzierten mehr Interleukin-4.
Die IL-22-Spiegel waren in beiden Läsionen erhöht.

$\mathrm{Um} \mathrm{zu}$ testen, ob unspezifische oder antigenspezifische T-Zell-Reaktionen vorherrschen, wurde ein PatchTest mit dem Hauptantigen der Hausstaubmilbe durchgeführt. Bei zwei sensibilisierten Patienten wurde dadurch ein atopisches Ekzem mit überwiegender TH2-Antwort ausgelöst. Die TH2-Zellen waren zum großen Teil antigenspezifisch. Neue Psoriasisläsionen (Köbner-Phänomen) traten nicht auf.

Diese Beobachtungen sprechen gegen eine intrinsische Epithelstörung als pathogenetische Basis von Psoriasis und atopischer Dermatitis, schreiben die Studienautoren unter Leitung von Kilian Eyerich. Sie vermuten eine kausale Rolle von verschiedenen T-Zell-Subtypen, die durch krankheitsspezifische Antigene getriggert in die Haut einwandern.

Die Unterschiede zwischen Psoriasis und atopischer Dermatitis machten sich auch in der Therapie der doppelt erkrankten Patienten bemerkbar. Unter dem TNF- $\alpha$-Hemmer Infliximab verschwanden bei einem Mann zwar die Psoriasisläsionen, dafür begann sein Ekzem zu blühen.

Fazit: Die Pathogenese von Psoriasis und atopischer Dermatitis scheint durch unterschiedliche, antigenspezifische Subgruppen von T-Zellen bestimmt zu werden. Bei der Psoriasis sind die auslösenden Antigene noch nicht identifiziert.

Dr. Beate Schumacher

Eyerich S et al. Mutual antagonism of T cells causing psoriasis and atopic eczema. N Engl J Med 2011; 365: 231-8

\title{
Was Nahrungsmittelallergien kosten
}

\section{Etwa 4 bis $6 \%$ der Kinder und 1 bis $2 \%$ der Erwachsenen in den USA haben Nahrungsmittelallergien. Jetzt wurde abgeschätzt, welche Kosten für ihre Behandlung dort jährlich anfallen.}

$\mathrm{D}$ ie Kalkulation der durch Allergien gegen Nahrungsmittel verursachten direkten und indirekten Kosten erfolgte in einem Bottom-up-Ansatz aus der gesellschaftlichen Perspektive. Dazu wurden retrospektiv die Krankheitskosten pro Patient errechnet und mit den verfügbaren US-amerikanischen Prävalenzdaten für Nahrungsmittelallergien multipliziert. Die direkten Kosten der Behandlungen von nahrungsmittelallergisch bedingten Reaktionen wurden aus Datenbanken der Jahre 2006 und 2007 ermittelt. Die indirekten Kosten wurden abgeschätzt anhand des krankheitsbedingten Produktionsausfalls.

Für 2007 ergaben sich direkte Kosten von 225 Mio. US-Dollar und indirekte Kosten von 115 Mio. US-Dollar. Das Aufsuchen von Behandlungszentren/
Arztpraxen durch die Patienten machte $52,5 \%$ der direkten Kosten aus. Die andere Hälfte entstand durch Behandlungen in Notfallambulanzen (20\%), in Krankenhäusern (11,8\%), Hausbesuche $(3,9 \%)$ sowie durch den Einsatz von Krankenwagen (3,0\%) und Adrenalininjektoren (8,7\%). Da diese Zahlen z. B. durch Ungenauigkeiten in Diagnostik bzw. Kodierungen konservative Schätzungen darstellten, wurden auch probabilistische Sensitivitätsanalysen unter Einbeziehung von Variablen verschiedener medizinischer Kosten sowie allgemeiner Variablen wie z. B. der durchschnittlichen Tagesverdienste durchgeführt. In diesen Berechnungen ergaben sich insgesamt 307 Mio. US-Dollar für die direkten und 203 Mio. US-Dollar für die indirekten Kosten.

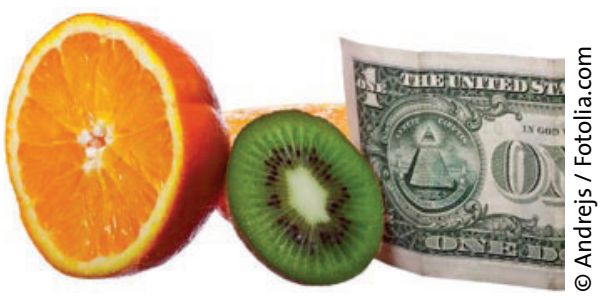

Teure Früchtchen - die Behandlung von Nahrungsmittelallergien schluckt in den USA jährlich eine halbe Mrd. US-Dollar.

Fazit: In den USA verursachen Reaktionen aufgrund einer Nahrungsmittelallergie jährliche Kosten von rund einer halben Milliarde US-Dollar. Mehr als die Hälfte der Ausgaben fielen auf die ambulanten Behandlungen. Die hier verursachten Kosten lagen im Durchschnitt aber deutlich niedriger als bei Behandlungen im Krankenhaus bzw. im Rahmen von Notaufnahmen.

Dr. Barbara Kreutzkamp

Patel DA et al. Estimating the economic burden of food-induced allergic reactions and anaphylaxis in the United States. J Allergy Clin Immunol 2011; 128: 110-5.e5 